\title{
p16 Gene Transfer Induces Centrosome Amplification and Abnormal Nucleation Associated with Survivin Downregulation in Glioma Cells
}

\author{
Takeshi Takayasu $^{\mathrm{a}}$ Seiji Hama ${ }^{\mathrm{a}}$ Fumiyuki Yamasaki $^{\mathrm{a}}$ Taiichi Saito $^{\mathrm{a}}$ \\ Yosuke Watanabe $^{c}$ Ryo Nosaka ${ }^{a} K$ Kazuhiko Sugiyama ${ }^{b}$ Kaoru Kurisu ${ }^{a}$ \\ a Department of Neurosurgery, Graduate School of Biomedical and Health Sciences, Hiroshima University, and \\ ${ }^{b}$ Department of Clinical Oncology and Neuro-Oncology Program, Cancer Treatment Center, Hiroshima University \\ Hospital, Hiroshima, and ' Department of Neurosurgery, Matsuyama Red Cross Hospital, Ehime, Japan
}

\section{Key Words}

Glioma cell lines · p16 · Centrosome amplification .

Survivin · Cell cycle arrest

\begin{abstract}
Objective: In human glioma cells, p16 gene transfer induced G1/S arrest, increased radiosensitivity and abnormal nucleation (especially bi- and multinucleation). Survivin suppression caused G2/M arrest, radiosensitization and an increase in aneuploidy accompanied by centrosome amplification. Abnormal nucleation and aneuploidy represent chromosome instability (CIN), and it is well known that centrosome amplification leads to CIN. However, little has been reported that suggests that transferring $\mathrm{p} 16$ causes centrosome overduplication during the G1/S phase. Methods: The p16 gene was transferred into p16-null human glioma cell lines (U251MG and D54MG) using adenovirus with or without irradiation. Centrosome amplification was evaluated by immunofluorescence. We also investigated the DNA replication licensing factor CDT1, its inhibitor geminin and survivin expression as regulators of chromosomal segregation. $\boldsymbol{R e}$ sults: $p 16$ gene transfer with radiation initiated the greatest degree of centrosome overduplication. CDT1 showed low levels, geminin was unchanged and survivin decreased in Ax-hp16-infected cells with radiation. Those changes of fac-
\end{abstract}

tors affecting DNA licensing or chromosomal segregation might contribute to CIN. Conclusion: p16 transfer caused centrosome amplification even in $\mathrm{G} 1 / \mathrm{S}$ phase-arrested cells. This suggests that p16 is involved in abnormal nucleation and radiosensitization in human glioma cells.

C 2015 S. Karger AG, Basel

\section{Introduction}

Malignant human gliomas diffusely invade surrounding normal brain tissue, making surgical removal of the entire tumor difficult. The clinical effectiveness of radiotherapy has so far been limited; therefore, this research aimed to explore how the radiosensitivity of glioma cells can be increased [1-3].

The p16 gene is located at chromosomal region 9p21, and its product is thought to prevent the progression of eukaryotic cells through G1 arrest in the cell cycle by binding to cyclin-dependent kinase (CDK) 4 and CDK6, and preventing them from forming an active complex with cyclin-D proteins [4-6]. The inactivation of p16 has been linked to the development of malignant gliomas. We previously demonstrated that adenovirus-mediated p16 gene transfer caused G1 arrest and enhanced the radiosensitivity of glioma cell lines [7]. The irradiated p16

\section{KARGER 125}

(c) 2015 S. Karger AG, Base

1015-2008/15/0821-0001\$39.50/0

E-Mail karger@karger.com

www.karger.com/pat
T. Takayasu

Graduate School of Biomedical and Health Sciences

Hiroshima University, 1-2-3, Kaumi, Minami-ku

Hiroshima City, Hiroshima 734-8551 (Japan)

E-Mail ttakayasu-nsu@umin.ac.jp 
gene-transferred cells had an increased frequency of abnormal nuclei (especially bi- and multinucleation) and exhibited nonapoptotic cell death. These data suggest that there is an unknown interaction between radiation and p16 that decreases cytokinesis and promotes abnormal nucleation.

Survivin, the smallest member in the family of inhibitor of apoptosis proteins, is a 142 -amino acid, $16.5-\mathrm{kDa}$ protein that inhibits apoptosis [8]. Survivin is involved in the spindle checkpoint and the regulation of proper chromosomal segregation during mitosis, thus it is also considered a chromosomal passenger protein $[9,10]$. The synthesis and degradation of survivin in normal tissues is modulated in a cell cycle-dependent manner; survivin levels increase during the $\mathrm{G} 1$ phase and peak in the G2/M phase [11]. Previously, we transfected human glioma cell lines with small interfering RNA to suppress survivin expression and then examined the radiosensitivity of the transfected cells. The transfected cells demonstrated G2/M cell-cycle arrest and extreme centrosome amplification accompanied by an increased degree of aneuploidy (chromosome instability) and mitotic cell death [12]. Thus, in our previous studies, we found that p16 gene expression or suppression of survivin expression led to G1/S or G2/M arrest, respectively, and induced nonapoptotic cell death accompanied by abnormal nucleation.

The licensing of DNA replication is one of the important events during the transition from the G1/S phase to the G2/M phase [13]. The DNA of the chromosomes is replicated during the $S$ phase, and the replicated chromosomes segregate into the two daughter cells during the $M$ phase of the cell cycle. Chromosomal replication is regulated such that it is initiated only once (during the S phase) in each cell cycle through a process called licensing. Among the licensing-related factors, cdc10-dependent transcript 1 (CDT1; positive regulator for DNA synthesis) and geminin (negative regulator for DNA synthesis) are the key regulatory factors. CDT1 helps in the formation of the prereplication complex by loading minichromosome maintenance proteins 2-7 onto chromatin [1418]; DNA polymerase binds to this complex and initiates DNA synthesis in the $S$ phase. Geminin negatively regulates the function of CDT1 and inhibits relicensing during the $\mathrm{G} 2 / \mathrm{M}$ phase. This process continues throughout the cell cycle to limit DNA replication to once per cycle. To maintain this process, the expression levels of CDT1 and geminin fluctuate at different stages of the cycle.

The centrosome is a major microtubule-organizing center in eukaryotic cells. Two centrosomes form spindle poles in mitosis. Thus, the proper duplication of centro- somes is essential for the formation of bipolar mitotic spindles and accurate chromosome segregation into two daughter cells during cytokinesis. Centrosome duplication is highly regulated and occurs once per cell cycle. When this control is disturbed, centrosome amplification (the presence of $>2$ centrosomes) arises, resulting in aberrant mitotic spindles and increased chromosome segregation errors, which lead to abnormal nucleation [12, 19-22].

The association between centrosome overduplication leading to chromosome instability (abnormal nucleation) and radiosensitivity has been well documented, but most of the documented mechanisms have been related to dysfunction of the G2/M phase [12, 19-21, 23]. However, we previously demonstrated that G1/S arrest induced by $\mathrm{p} 16$ gene expression also led to abnormal nucleation and enhanced radiosensitivity [7]. Thus, we hypothesized that p16 gene transfer can cause centrosome overduplication even during the $\mathrm{G} 1 / \mathrm{S}$ phase, resulting in abnormal nucleation. We also investigated the DNA replication licensing factor CDT1 and its inhibitor geminin as the key components for advancing the cell cycle from the G1/S phase to G2/M phase, as well as survivin expression levels in p16transferred cells.

\section{Materials and Methods}

Cells

U251MG cells [human glioma; p16, homozygous deletion; p53, mutated at codon 273 (CGT/CAT Arg/His)], D54MG cells (human glioma; p16, undetectable protein expression; p53, wild type) and A549 (human non-small cell lung cancer; p16, undetectable protein expression; p21, undetectable protein expression; p53, wild type) were used in the present study. These cells were obtained in the same manner as previously described $[7,12]$.

\section{Adenovirus Vectors}

The p16- or p21-expressing adenovirus vectors used here were also used in our previous studies [24, 25]. In brief, replicationdefective adenovirus vectors were based on the human adenovirus type 5 serotype. Recombinant adenovirus vectors containing the full-length human p16 gene (Ax-hp16) or p21 gene (Ax-hp21) were generated using the COS-TPC method. Exogenic p16 or p21 gene expression after Ax-hp16 or Ax-hp21 infection has been achieved in our previous studies [24, 25]. Control vectors consisted of recombinant adenoviruses containing an expression cassette without any genome (mock adenovirus vector; Ax-mock). In the present study, all gene transfers were performed using continuous infection without changing the culture medium until analysis, as was performed in previous experiments [7], in order to maintain adenovirus expression over 5-7 days [25]. As continuous adenovirus infection is particularly likely to cause direct cytotoxicity, we assessed the cytotoxicity of the vector alone using plating efficiency as the end point. Cytotoxicity became evident at multiplicity of infection (MOI) values between 5 and 10 in U251MG and D54MG
Takayasu/Hama/Yamasaki/Saito/ Watanabe/Nosaka/Sugiyama/Kurisu 
cells. In further preliminary experiments, we used MOI values of 6 in both U251MG and D54MG cells to assess radiosensitization (data not shown).

\section{Irradiation and Adenovirus Infection}

To obtain uniform and reproducible irradiation conditions, cells were trypsinized, counted, placed in a polypropylene tube and irradiated using a ${ }^{60} \mathrm{Co}$ source at a dose rate of $0.65 \mathrm{~Gy} \mathrm{~min}^{-1}$. Known numbers of cells were then replated in culture dishes and returned to the incubator. At about $12 \mathrm{~h}$ after irradiation, U251MG cells and D54MG cells were infected at an MOI of 6 .

\section{Immunofluorescence}

Cells grown in culture for 3-5 days were washed twice with PBS and collected by trypsinization. The culture medium and the PBS used for the first washing were collected and centrifuged together with the trypsinized cell suspension because some of the cells were detached from the bottom of the dish and floating, especially in the Ax-hp16-infected samples. The pellets were smeared on coverslips, and immediately coated with Tissue Capture (Cosmo Bio, Tokyo, Japan). The samples were fixed and permeabilized with $100 \%$ methanol for $10 \mathrm{~min}$ at $4{ }^{\circ} \mathrm{C}$, incubated with blocking solution (15\% bovine serum albumin in PBS) for $30 \mathrm{~min}$ and probed with antibodies for $1 \mathrm{~h}$ at $37^{\circ} \mathrm{C}$. The antibody used for immunostaining was mouse anti- $\gamma$-tubulin monoclonal antibody-conjugated Alexa Fluor 488 (sc-17788 AF488; Santa Cruz Biotechnology, Santa Cruz, Calif., USA). The samples were washed with TBS buffer and then counterstained with DAPI ( $4^{\prime}, 6^{\prime}$-diamidino-2-phenylindole). These experiments were repeated twice.

\section{Immunoblot Analysis}

On days $3(72 \mathrm{~h})$ and $5(120 \mathrm{~h})$ after infection with Ax-hp16 or Ax-mock, cell lysates from U251MG (mutant p53) and D54MG (wild-type p53) were extracted using $100 \mu$ of ice-cold lysis buffer (25 mM Tris- $\mathrm{HCl}, \mathrm{pH} 7.4,50 \mathrm{~mm} \mathrm{NaCl}, 0.5 \% \mathrm{Na}$ deoxycholate, $2 \%$ Nonidet P-40, $0.2 \%$ SDS, $1 \mathrm{~mm}$ phenylmethylsulfonyl fluoride and $50 \mu \mathrm{g} / \mathrm{ml}$ aprotinin). The lysates were boiled, size-fractionated through 6-15\% SDS-PAGE, and transferred onto a polyvinylidene difluoride membrane (Millipore, Bedford, Mass., USA). The membrane was blocked with TBS containing $0.05 \%$ Tween 20 and $0.3 \%$ skimmed milk, and was then probed with rabbit anti-human survivin monoclonal antibody (No. 2808; Cell Signaling, Danvers, Mass., USA), rabbit anti-human CDT1 monoclonal antibody (No. 8064; Cell Signaling), rabbit anti-human geminin polyclonal antibody (No. 5165; Cell Signaling), mouse anti-cyclin E1 monoclonal antibody (No. 4129S, Cell Signaling) or anti- $\beta$-actin monoclonal antibody (G043; Applied Biological Materials, Richmond, B.C., Canada). Chemiluminescent detection was performed with ECL Western blotting detection reagents (Amersham, Arlington Heights, Ill., USA). These experiments were repeated at least twice.

\section{Evaluation of the Number of Centrosomes}

The number of centrosomes in U251MG and D54MG cells was analyzed by immunofluorescent staining for $\gamma$-tubulin on days 3 (72 h) and 5 (120 h) after infection with Ax-hp16, Ax-hp21 or Axmock with or without irradiation (4 Gy). The round spots stained with anti- $\gamma$-tubulin antibody were recognized as centrosomes and scored per tumor cell. Artifacts (nonspecific staining) are evident because of the diverse cellular debris that accompanied cell death. Centrosome overduplication could be recognized in some of these

p16 Gene Transfer Induces Centrosome Amplification in Glioma Cells dead cells but those in which overduplication was difficult to recognize were excluded from the counts. More such cells were observed in the Ax-hp16-infected samples; thus, there is a possibility that centrosome overduplication was underestimated in the Ax-hp16-infected samples. The results are presented as the percentage of cells containing more than two centrosomes, with a minimum of 200 cells being scored. Each experiment was repeated at least twice.

\section{Results}

In preliminary experiments, we confirmed that U251MG cells or D54MG cells infected with Ax-hp16 were significantly more radiosensitive than noninfected or Ax-mock-infected cells [7]. This trend was similar to that observed in Ax-hp21-infected U251MG cells [7]. The most effective radiosensitization with less direct cytotoxicity was achieved by using an MOI value of 6 for both U251MG and D54MG cells.

\section{Number of Centrosomes in U251MG and D54MG \\ Cells}

We evaluated the number of centrosomes by immunofluorescence. Figure 1 shows representative cells containing normal numbers of centrosomes or centrosome amplification. Figure 2 illustrates that p16 or p 21 transfer in both cell types results in centrosome overduplication. In D54MG cells, the proportion of cells with centrosome amplification induced by p16 was not apparently higher than in other control cells on day 3. Additionally, most samples showed centrosome numbers of 3-4 (mild amplification), and less than half of the samples showed centrosome numbers of 5 or more (marked amplification). In contrast, irradiated Ax-hp16-infected D54MG cells contained a greater number of cells with centrosome amplification by day 5, and more than half of these showed marked amplification. This result indicates that p16 transfer causes an increase in the number of centrosomes in both cell lines, but the increase in U251MG cells was more pronounced and occurred earlier than in D54MG cells. These trends were confirmed in repeated experiments.

\section{CDT1, Geminin and Cyclin E Expression}

To confirm the status of the DNA synthesis cycle in Ax-hp16-infected cells, we investigated CDT1 and geminin expression levels as the licensing factors. The expression of CDT1 was decreased in both Ax-hp16- and Axmock-infected cells, but the reduction was greater in the Ax-hp16-infected cells. The reduction in CDT1 expression in even the mock-infected cells suggests that the continuous adenovirus infection conditions used for the gene 

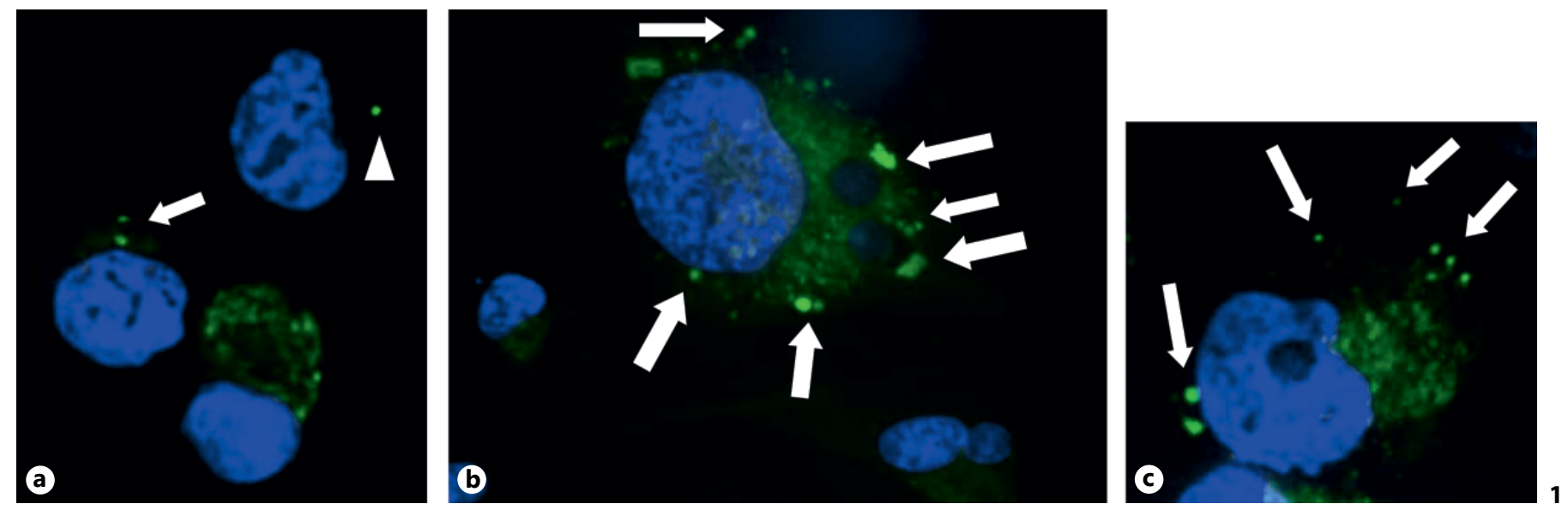

Fig. 1. Immunofluorescence analysis on day $3(72 \mathrm{~h})$ after transfection. Cells were immunostained for centrosomes (green dot) using anti- $\gamma$-tubulin and counterstained for DNA with $4^{\prime}, 6^{\prime}$-diamidino2-phenylindole (blue). Representative cells are shown. a Ax-mock-infected D54MG cells without radiation, containing one (arrow head) or two centrosomes (arrow). b Ax-hp16-infected U251MG cells with radiation, harboring multiple centrosomes (arrows) and showing multinucleation. c Ax-hp16-infected D54MG cells, without radiation, containing 8 centrosomes (arrows).

Fig. 2. The percentage of cells containing more than two centrosomes was determined by immunostaining. Comparison of the percentage of U251MG and D54MG cells containing more than two centrosomes on days $3(72 \mathrm{~h} ; \mathbf{a})$ and $5(120 \mathrm{~h} ; \mathbf{b})$ after Ax-mock or Ax-hp16 or Ax-hp21 infection with or without irradiation. Axhp16-infected cells and Ax-hp21-infected cells, especially with radiation, contain a higher proportion of cells with centrosome amplification.

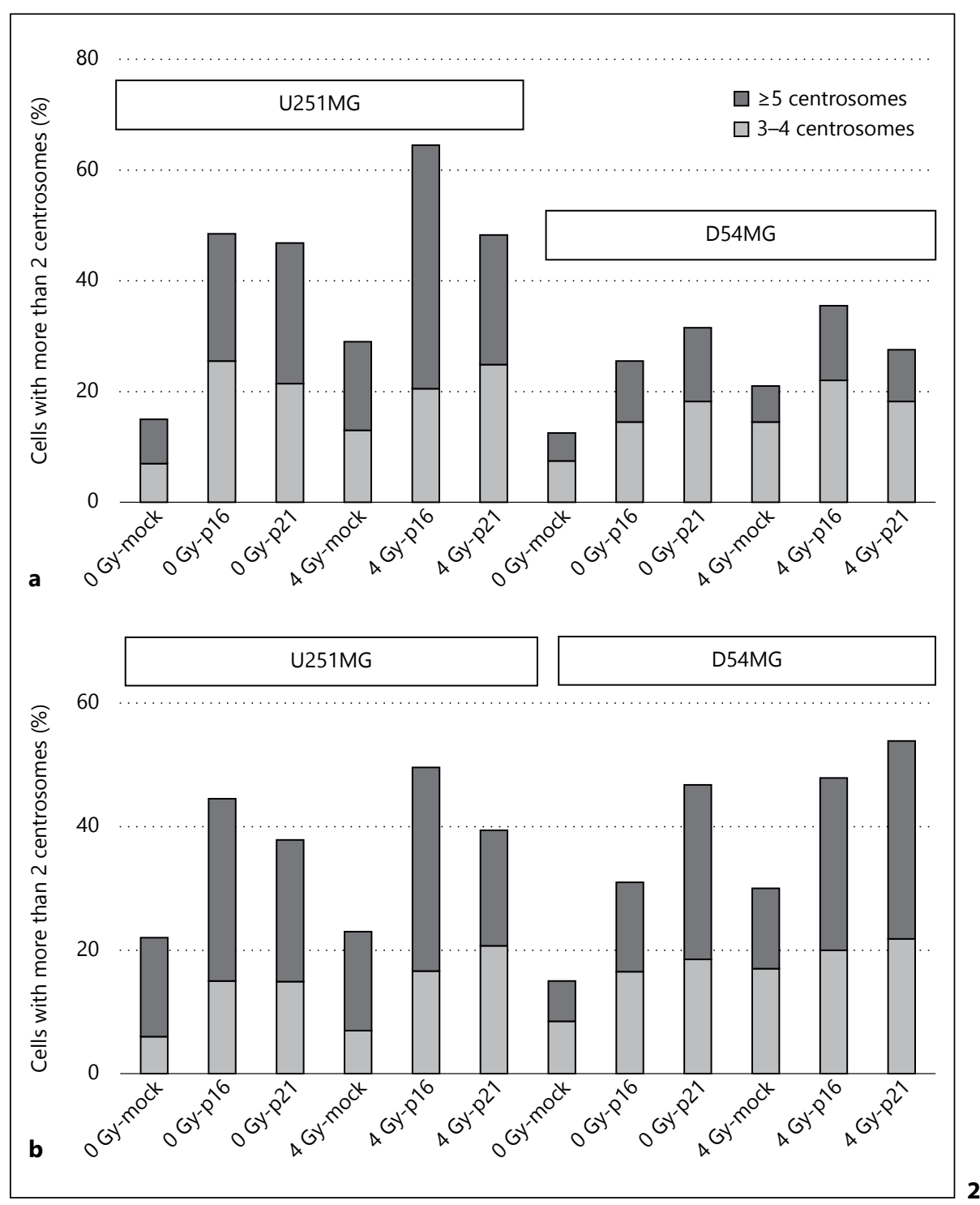


Fig. 3. Western blot analyses of CDT1, geminin, cyclin $\mathrm{E}$ and survivin expression in U251MG and D54MG cells at 72 and $120 \mathrm{~h}$ (on days 3 and 5) after infection with Ax-mock or Ax-hp16 with or without irradiation. Ax-hp16-infected cells show a low level of CDT1 and an unchanged level of geminin. Cyclin E expression (on day 3) is not changed by Ax-hp16 infection. Survivin expression is downregulated by $\mathrm{p} 16$ transfection. $\beta$-Actin serves as an internal loading control.

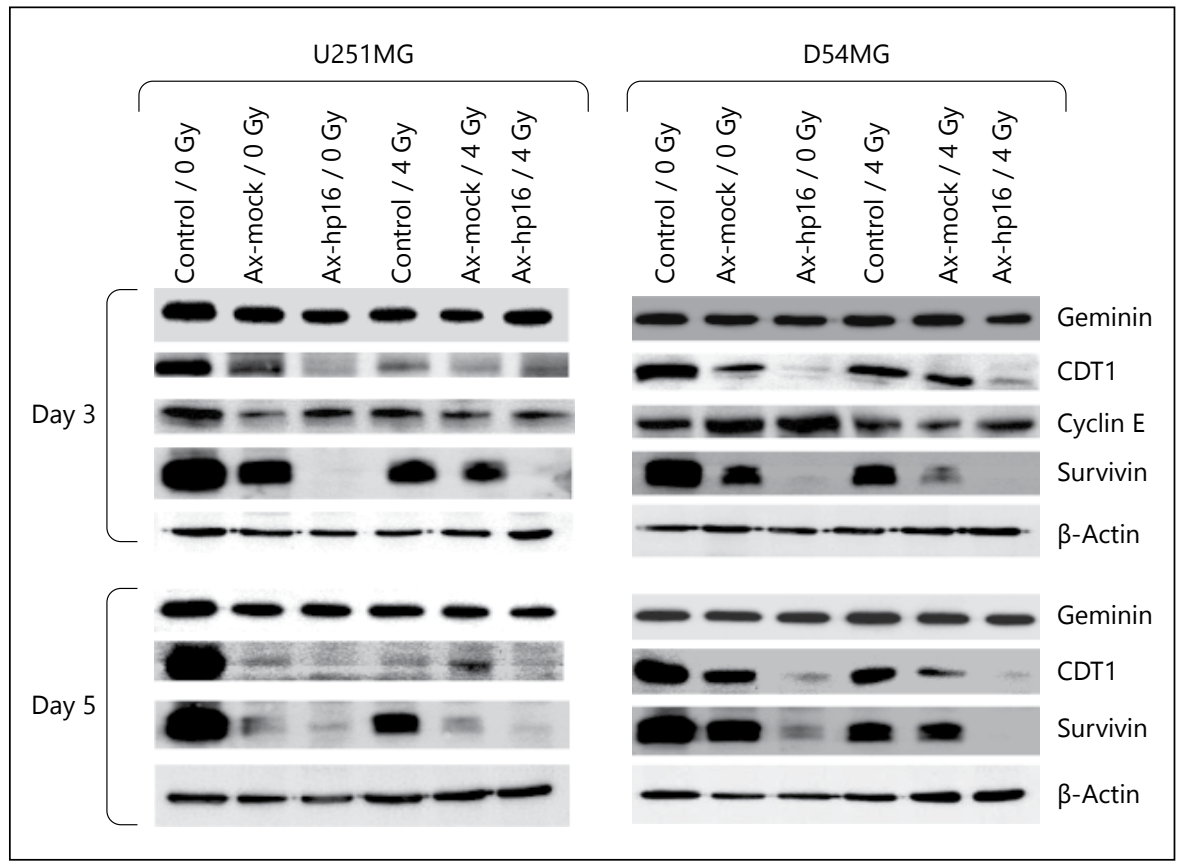

transfer may be responsible for some of the reduction. The reduction was even more pronounced in U251MG cells, which are thought to be more easily affected by virus compared to D54MG cells. Geminin expression was similar in both Ax-hp16-infected and control cells (fig. 3). We also examined cyclin E expression, because the CDK2cyclin E complex affects both centrosome duplication and DNA synthesis. Cyclin E levels were not different in Ax-hp16-infected cells and control cells (fig. 3).

\section{p16 Gene Transfer Downregulates Survivin Expression}

We investigated survivin expression in p16-transferred U251MG and D54MG cells (fig. 3). Survivin expression decreased even in Ax-mock-infected cells (especially U251MG cells), which might also be due to the direct cytotoxicity of adenovirus infection. Survivin was not expressed in Ax-hp16-infected cells but was expressed in noninfected or Ax-mock-infected cells, suggesting that p16 expression was directly involved in the reduction in survivin expression.

\section{Discussion}

Here we have shown that p16 gene transfer initiated centrosome overduplication after irradiation in glioma cell lines. The response to p16 transfer differed between U251MG and D54MG glioma lines. By day 3, centrosome amplification was evident in U251MG cells, but was not observed in D54MG cells. However, as the centrosome number in D54MG cells increased, the difference in centrosome amplification between U251MG and D54MG was no longer apparent by day 5 . This might be dependent on the cells' p53 status. It has been reported that the absence of p53 can initiate centrosome duplication [22]. Our previous study using survivin suppression showed similar results in that centrosome amplification and radiosensitivity were enhanced in p53-mutated cells [12].

In normal eukaryotic cells, centrosome duplication is initiated in the late G1 phase of the cell cycle and completed during the $\mathrm{G} 2$ phase in coordination with DNA synthesis. p16 inhibits CDK4 and CDK6, regulates the phosphorylation of $\mathrm{pRb}$, and leads to arrest at the G1/S transition [7, 26]. Using flow cytometry, we previously showed that the DNA content of Ax-hp16-infected cells was $2 n$, showing G1 arrest (online suppl. fig. 1; for all online suppl. material, see www.karger.com/doi/10.1159/000368196). Cells with a dysfunctional G2/M phase (mitosis failure) have CIN associated with centrosome overduplication [12, 19-21, 23]. In this study, we have explored the question of whether the previously observed centrosome overduplication could also occur in G1/S-arresting cells following p16 overexpression and irradiation.

Uncoupled centrosome duplication and DNA replication cycles have been observed previously [27]; the absence of p53 may initiate centrosome duplication much 
earlier in the cell cycle [22], or a defect in checkpoint controls at G1/S or G2/M can cause dissociation of the centrosome duplication cycle from cell cycle events [28]. However, little has been reported about an association between G1/S arrest induced by p16 gene transfer and centrosome duplication [29].

Three pathways to supernumerary centrosomes in cancer cells have been postulated: (1) the centrosome accumulation pathway due to polyploidization events is related to cytokinesis failure with or without normal DNA replication, and results in the accumulation of normally replicated centrosomes; (2) in the centrosome overreplication pathway, cells arrested in the S or G2 phases undergo multiple rounds of templated centriole duplication, leading to functional overduplicated centrosomes, and (3) in the de novo centriole formation pathway, new centrioles are assembled via centriole satellites [30]. It appears that the mechanisms of centrosome overduplication in survivin downregulated glioma cells [12] and Axhp16-infected glioma cells can be ascribed to the centrosome accumulation pathway and the centrosome overreplication pathway, respectively, because the former are arrested in the G2/M phase and accompanied by polyploidy, and the latter are arrested in the G1/S phase.

In normal cells, a mature centrosome consists of paired centrioles in orthogonal configuration. Centrosome duplication begins with separation of the two centrioles. The nucleation of daughter centrioles occurs at the base of the parental centrioles, and daughter centrioles subsequently elongate and mature. This centriole disengagement itself licenses centrosomes for duplication and occurs at the end of mitosis [21, 30,31]. Thus, the centrosomes in the Ax-hp16-infected cells, arrested in the G1/S phase, were ready for replication.

The coupling of centrosome duplication and DNA synthesis is, at least in part, achieved by the CDK2-cyclin E complex, which induces S-phase entry. In the present study, the expression of cyclin $\mathrm{E}$ was not downregulated after p16 gene expression, neither with nor without irradiation; thus, cyclin $\mathrm{E}$ has the capacity to initiate both centrosome and DNA duplication. Therefore, the Ax-hp16infected cells were blocked from progression to DNA duplication (S phase) by inactivation of CDK4/6, but maintained the capability for centrosome duplication as described above, which probably dissociated the centrosome duplication cycle from DNA replication.

To examine the status of DNA synthesis in Ax-hp16infected cells, we investigated CDT1 and geminin expression levels as the licensing factors, finding low levels of CDT1 and unchanged levels of geminin. These results in- dicate that CDT1 started to decrease as geminin synthesis began. These results suggest that Ax-hp16-infected glioma cells contained already licensed DNA and were blocked from progression to the S phase by inactivation of CDK4/6. However, in our previous paper, flow cytometry analysis showed that the distribution of histograms around DNA content of $2 n$ and $4 n$ was wider in the Ax-hp16-infected cells as compared to control cells (online suppl. fig. 1) [7], which suggests that the chromosomes of Ax-hp16-infected cells were partially replicated despite being arrested in the G1/S phase, thus resulting in abnormal nucleation (incomplete blocking of DNA synthesis; somewhat unstable).

Centrosome amplification leads to CIN [21, 27, 32, 33]. CIN is caused by mitotic or cytokinetic failure. In our previous paper, survivin downregulation induced supernumerary centrosomes without cytokinesis, resulting in polyploid cells. Ax-hp16-infected cells, arrested at the G1/S boundary, did not show polyploidy but showed multinucleation, representing another kind of CIN. As described above, the cancer cells could overduplicate centrosomes even if arrested in the $\mathrm{S}$ phase (centrosome overreplication pathway). The findings of this study suggest that while Ax-hp16-infected cells did not undergo cytokinesis, they contained partially licensed DNA and overduplicated centrosomes, leading to CIN due not to polyploidy, but rather to abnormal nucleation (i.e. micronucleation, bi- and multinucleation).

To investigate whether arrest in the G1/S phase was caused by factors other than p16-induced centrosome amplification, we also evaluated the number of centrosomes in Ax-hp21-infected cells (fig. 2).p21 transfection caused similar centrosome amplification and was expected to induce CIN and radiosensitivity, since our previous study showed that both Ax-hp16 and Ax-hp21 infection increased radiation-induced cell killing [7]. Those results suggested that the induction of CIN and radiosensitivity were not due to the molecular property of overexpressed p16, but instead to the cell-cycle arrest at G1/S.

To confirm whether the centrosome overduplication induced by Ax-hp16 infection was specific for human glioma cell lines, we also observed centrosome numbers in other p16-null human cancer cell lines infected with Axhp16, the non-small cell lung cancer cell line A549 (p53, wild type). In previous experiments, we confirmed that p16 gene transfer increased radiosensitivity via nonapoptotic cell killing in A549 [7]. As shown in figure 4, centrosome amplification was also observed in p16-transferred A549 cells. Interestingly, similar to D54MG cells, centrosome overduplication in Ax-hp16-infected A549 cells without radiation was delayed and developed by day 5 ,
Takayasu/Hama/Yamasaki/Saito/ Watanabe/Nosaka/Sugiyama/Kurisu 
Fig. 4. The percentage of cells with centrosome amplification in A549 cells after Axmock or Ax-hp16 infection with or without irradiation. Centrosome amplification was observed in p16-transferred A549 cells.

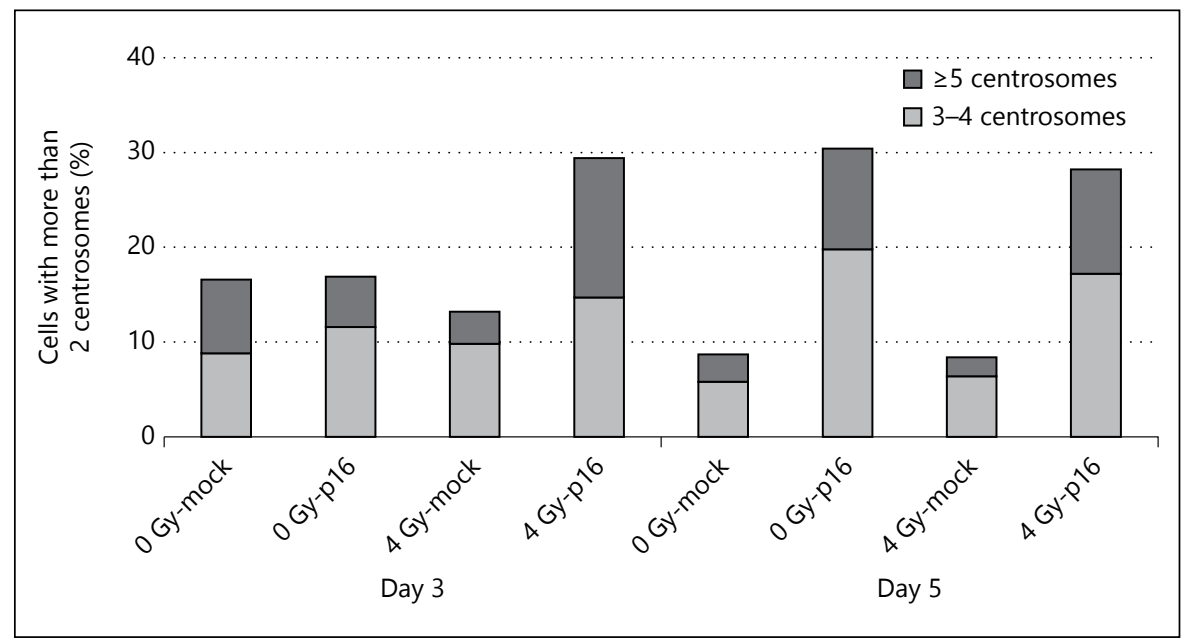

which also might be dependent on the p53 wild-type state. Those results suggested that p16 transfer could confer CIN not only in glioma cells, but also to other kinds of tumor cells.

In our present study, survivin expression in Ax-hp16infected cells decreased. Hu et al. [34] also reported that p16 transfer downregulated survivin expression through inactivation of the phosphatidylinositol 3-kinase/Akt pathway in hepatocellular carcinoma cells. Survivin mediates the proper targeting of chromosomal passenger proteins to kinetochores and stabilizes the microtubules, contributing to bipolar spindle formation [11, 35]. Moreover, it was reported that survivin regulates not only the G2/M phase, but also G1/S progression [34-36]. Thus, survivin downregulation might induce chromosomal segregation errors in p16-transferred cells, resulting in CIN. In other words, both amplified centrosomes and downregulated survivin probably contribute to CIN in cells in the G1/S phase. We did not investigate the molecular and genetic background of survivin downregulation after p16 transfer in these experiments; thus, further investigation will be needed.

The findings from this study did not clarify the association between centrosome amplification and radiationinduced cell death (radiosensitivity). Figure 2 shows that centrosome overduplication was observed in less than $70 \%$ of tumor cells even after the p16 transfer, meaning that more than $30 \%$ of tumor cells might be radioresistant. This radioresistant population probably included so-called tumor stem cells that are thought to make such tumors intractable. Therefore, further studies, perhaps using a xenograft transplantation system for tumor stem cells, will be needed to clarify the interaction between centrosome overduplication (CIN), survivin and radiation sensitivity.

In conclusion, the present results suggest that p16 overexpression leads to centrosome amplification in human glioma cells. Although centrosome overduplication and chromosome instability (abnormal nucleation) has been studied mainly in the G2/M phase, this study suggests that the G1/S phase, p16 and survivin are also important. Centrosome overduplication and chromosome instability were thought to be related to radiosensitivity; thus, studies of p16/survivin will further elucidate the mechanisms of centrosome duplication/CIN, resulting in the development of strategies for improving radiation sensitivity.

\section{Acknowledgements}

This study was supported in part by Grants-in-Aid for Scientific Research (C) No. 23592126 and Young Scientists (B) No. 24791503 from the Ministry of Education, Culture, Sports, Science and Technology of Japan. The work was carried out at the Analysis Center of Life Science, Natural Science Center for Basic Research and Development, Hiroshima University.

References

1 Anandharaj A, Cinghu S, Park WY: Rapamycin-mediated mTOR inhibition attenuates survivin and sensitizes glioblastoma cells to radiation therapy. Acta Biochim Biophys Sin (Shanghai) 2011;43:292-300.

$\checkmark 2$ Lanvin O, Monferran S, Delmas C, Couderc B, Toulas C, Cohen-Jonathan-Moyal E: Radiation-induced mitotic cell death and glioblastoma radioresistance: a new regulating pathway controlled by integrin-linked kinase, hypoxia-inducible factor 1 alpha and survivin in U87 cells. Eur J Cancer 2013;49:2884-2891. 
3 Scaringi C, Enrici RM, Minniti G: Combining molecular targeted agents with radiation therapy for malignant gliomas. Onco Targets Ther 2013;6:1079-1095.

$\checkmark 4 \mathrm{He} \mathrm{J}$, Allen JR, Collins VP, Allalunis-Turner MJ, Godbout R, Day RS 3rd, James CD: CDK4 amplification is an alternative mechanism to p16 gene homozygous deletion in glioma cell lines. Cancer Res 1994;54:58045807.

5 Nobori T, Miura K, Wu DJ, Lois A, Takabayashi K, Carson DA: Deletions of the cyclin-dependent kinase-4 inhibitor gene in multiple human cancers. Nature 1994;368: 753-756.

-6 Tam SW, Shay JW, Pagano M: Differential expression and cell cycle regulation of the cyclin-dependent kinase 4 inhibitor p16Ink4. Cancer Res 1994;54:5816-5820.

7 Hama S, Matsuura S, Tauchi H, Yamasaki F, Kajiwara Y, Arita K, Yoshioka H, Heike Y, Mandai K, Kurisu K: p16 gene transfer increases cell killing with abnormal nucleation after ionising radiation in glioma cells. $\mathrm{Br} \mathrm{J}$ Cancer 2003;89:1802-1811.

8 Altieri DC: The molecular basis and potential role of survivin in cancer diagnosis and therapy. Trends Mol Med 2001;7:542-547.

-9 Adams RR, Maiato H, Earnshaw WC, Carmena M: Essential roles of Drosophila inner centromere protein (INCENP) and aurora B in histone $\mathrm{H} 3$ phosphorylation, metaphase chromosome alignment, kinetochore disjunction, and chromosome segregation. J Cell Biol 2001;153:865-880.

10 Li F, Ambrosini G, Chu EY, Plescia J, Tognin S, Marchisio PC, Altieri DC: Control of apoptosis and mitotic spindle checkpoint by survivin. Nature 1998;396:580-584.

-11 Mita AC, Mita MM, Nawrocki ST, Giles FJ: Survivin: key regulator of mitosis and apoptosis and novel target for cancer therapeutics. Clin Cancer Res 2008;14:5000-5005.

12 Saito T, Hama S, Izumi H, Yamasaki F, Kajiwara Y, Matsuura S, Morishima K, Hidaka T, Shrestha P, Sugiyama K, Kurisu K: Centrosome amplification induced by survivin suppression enhances both chromosome instability and radiosensitivity in glioma cells. $\mathrm{Br} \mathrm{J}$ Cancer 2008;98:345-355

13 Xouri G, Dimaki M, Bastiaens PI, Lygerou Z: Cdt1 interactions in the licensing process: a model for dynamic spatiotemporal control of licensing. Cell Cycle 2007;6:1549-1552.
14 Ballabeni A, Zamponi R, Moore JK, Helin K, Kirschner MW: Geminin deploys multiple mechanisms to regulate Cdt1 before cell division thus ensuring the proper execution of DNA replication. Proc Natl Acad Sci U S A 2013;110:E2848-E2853.

15 Kisielewska J, Blow JJ: Dynamic interactions of high Cdt1 and geminin levels regulate $\mathrm{S}$ phase in early Xenopus embryos. Development 2012;139:63-74.

16 Shrestha P, Saito T, Hama S, Arifin MT, Kajiwara Y, Yamasaki F, Hidaka T, Sugiyama K, Kurisu K: Geminin: a good prognostic factor in high-grade astrocytic brain tumors. Cancer 2007;109:949-956.

17 Wong PG, Glozak MA, Cao TV, Vaziri C, Seto E, Alexandrow M: Chromatin unfolding by Cdt1 regulates MCM loading via opposing functions of $\mathrm{HBO} 1$ and HDAC11-geminin. Cell Cycle 2010;9:4351-4363.

18 Xouri G, Lygerou Z, Nishitani H, Pachnis V, Nurse P, Taraviras S: Cdt1 and geminin are down-regulated upon cell cycle exit and are over-expressed in cancer-derived cell lines. Eur J Biochem 2004;271:3368-3378.

19 Sato N, Mizumoto K, Nakamura M, Tanaka M: Radiation-induced centrosome overduplication and multiple mitotic spindles in human tumor cells. Exp Cell Res 2000;255:321-326.

20 Sato N, Mizumoto K, Nakamura M, Ueno H, Minamishima YA, Farber JL, Tanaka M: A possible role for centrosome overduplication in radiation-induced cell death. Oncogene 2000;19:5281-5290.

21 Fukasawa K: p53, cyclin-dependent kinase and abnormal amplification of centrosomes. Biochim Biophys Acta 2008;1786:15-23.

22 Fukasawa K, Choi T, Kuriyama R, Rulong S, Vande Woude GF: Abnormal centrosome amplification in the absence of p53. Science 1996;271:1744-1747.

23 Ganem NJ, Godinho SA, Pellman D: A mechanism linking extra centrosomes to chromosomal instability. Nature 2009;460:278-282.

24 Yamasaki F, Hama S, Yoshioka H, Kajiwara Y, Yahara K, Sugiyama K, Heike Y, Arita K, Kurisu K: Staurosporine-induced apoptosis is independent of p16 and p21 and achieved via arrest at G2/M and at G1 in U251MG human glioma cell line. Cancer Chemother Pharmacol 2003;51:271-283.
25 Hama S, Heike Y, Naruse I, Takahashi M, Yoshioka H, Arita K, Kurisu K, Goldman CK, Curiel DT, Saijo N: Adenovirus-mediated p16 gene transfer prevents drug-induced cell death through G1 arrest in human glioma cells. Int J Cancer 1998;77:47-54.

26 Sherr CJ: Cancer cell cycles. Science 1996;274: 1672-1677.

27 Lingle WL, Lukasiewicz K, Salisbury JL: Deregulation of the centrosome cycle and the origin of chromosomal instability in cancer. Adv Exp Med Biol 2005;570:393-421.

28 D'Assoro AB, Busby R, Suino K, Delva E, Almodovar-Mercado GJ, Johnson H, Folk C, Farrugia DJ, Vasile V, Stivala F, Salisbury JL: Genotoxic stress leads to centrosome amplification in breast cancer cell lines that have an inactive $\mathrm{G} 1 / \mathrm{S}$ cell cycle checkpoint. Oncogene 2004;23:4068-4075.

29 Balczon R, Bao L, Zimmer WE, Brown K, Zinkowski RP, Brinkley BR: Dissociation of centrosome replication events from cycles of DNA synthesis and mitotic division in hydroxyurea-arrested Chinese hamster ovary cells. J Cell Biol 1995;130:105-115.

30 Pihan GA: Centrosome dysfunction contributes to chromosome instability, chromoanagenesis, and genome reprograming in cancer. Front Oncol 2013;3:277.

31 Nigg EA: Cell biology: a licence for duplication. Nature 2006;442:874-875.

32 Fang X, Zhang P: Aneuploidy and tumorigenesis. Semin Cell Dev Biol 2011;22:595-601.

-33 Vitre BD, Cleveland DW: Centrosomes, chromosome instability (CIN) and aneuploidy. Curr Opin Cell Biol 2012;24:809-815.

34 Hu H, Li Z, Chen J, Wang D, Ma J, Wang W, Li J, Wu H, Li L, Wu M, Qian Q, Su C: P16 reactivation induces anoikis and exhibits antitumour potency by downregulating Akt/ survivin signalling in hepatocellular carcinoma cells. Gut 2011;60:710-721.

35 Wiedemuth R, Klink B, Topfer K, Schrock E, Schackert G, Tatsuka M, Temme A: Survivin safeguards chromosome numbers and protects from aneuploidy independently from p53. Mol Cancer 2014;13:107.

-36 Bian K, Fan J, Zhang X, Yang XW, Zhu HY, Wang L, Sun JY, Meng YL, Cui PC, Cheng SY, Zhang J, Zhao J, Yang AG, Zhang R: MicroRNA-203 leads to G1 phase cell cycle arrest in laryngeal carcinoma cells by directly targeting survivin. FEBS Lett 2012; 586:804-809. 